\section{Prevención de neumonía asociada a ventilación con paquete de verificación en la Unidad de Cuidados Intensivos. Estudio piloto}

Hernández-Orozco HG ${ }^{1}$, Castañeda-Narváez $\mathrm{JL}^{1}$, Lucas-Reséndiz $\mathrm{ME}^{2}$, RosasRuiz $\mathrm{A}^{3}$, Aparicio-Santiago $\mathrm{GL}^{3}$, Zárate-Castañón $\mathrm{P}^{4}$, Camacho-Soto $\mathrm{SA}^{5}$

\begin{abstract}
Resumen
INTRODUCCIÓN: los paquetes de verificación son una estrategia efectiva para la prevención de neumonías asociadas a ventilador.

OBJETIVO: determinar la viabilidad y beneficios de aplicar un paquete de verificación para prevenir neumonías asociadas a ventilador.

MATERIAL Y MÉTODOS: estudio piloto transversal, ambispectivo realizado del 2011 al 2013, determinando la tasa de neumonías asociadas a ventilador por 1,000 días ventilador en ese periodo y aplicando a partir del 2012 un paquete de verificación, para prevenir neumonías asociadas a ventilador en niños con ventilación mecánica en la Unidad de Cuidados Intensivos Pediátricos.
\end{abstract}

RESULTADOS: el cumplimiento de los parámetros evaluados mediante el paquete de prevención de neumonías asociadas a ventilador implementado en 2012 fue de entre 66 y 100\%, con una disminución en la incidencia de neumonías asociadas a ventilador de $47 \%$ en el periodo 2011 a 2012 y de 69\% en 2013, con tasas de neumonía asociada a ventilador de $13.85,7.29$ y 4.3 neumonías por 1,000 días ventilador, respectivamente.

CONCLUSIONES: el uso de paquete de verificación para prevención de neumonías es aplicable y efectivo para disminuir estas infecciones asociadas a la atención de la salud. Implica el compromiso y trabajo multidisciplinario de todo el servicio, así como un programa para llevar a cabo esta estrategia, por lo cual se planea su aplicación en todas las unidades de cuidados intensivos de la institución.

PALABRAS CLAVE: neumonía asociada a ventilador, paquete de verificación, prevención, pediatría, prevención efectiva.

Acta Pediatr Mex. 2016 Nov;37(6):322-327.

\section{Pilot study, VAP prevention using a bundle in the Intensive Care Unit.}

Hernández-Orozco HG ${ }^{1}$, Castañeda-Narváez $\mathrm{JL}^{1}$, Lucas-Reséndiz $\mathrm{ME}^{2}$, RosasRuiz $\mathrm{A}^{3}$, Aparicio-Santiago $\mathrm{GL}^{3}$, Zárate-Castañón $\mathrm{P}^{4}$, Camacho-Soto $\mathrm{SA}^{5}$

\section{Abstract}

INTRODUCTION: Use of verification bundles an effective strategy for the prevention of ventilator-associated pneumonia (VAP).
${ }^{1}$ Médico adscrito al Departamento de Infectología. ${ }^{2}$ Maestro en Salud Pública.

${ }^{3}$ Enfermera.

${ }^{4}$ Jefe de Servicio de Unidad de Cuidados Intensivos. ${ }^{5}$ Medico pasante de Servicio Social.

Instituto Nacional de Pediatría, México.

Recibido: 10 de octubre del 2015

Aceptado: 11 de mayo del 2016

Correspondencia

Dra. Hilda Gpe. Hernández-Orozco

wuzhi1916@gmail.com

Este artículo debe citarse como Hernández-Orozco HG, Castañeda-Narváez JL, LucasReséndiz ME, Rosas-Ruiz A, Aparicio-Santiago GL, Zárate-Castañón $\mathrm{P}$ et al. Prevención de neumonía asociada a ventilación con paquete de verificación en la Unidad de Cuidados Intensivos. Estudio piloto. Acta Pediatr Mex. 2016;37(6):322-327. 
OBJECTIVE: To determine the feasibility and benefits of applying a verification bundles to prevent VAP.

METHODS: Cross, ambispective pilot study from 2011 to 2013 determining the rate of ventilator-associated pneumonia per 1,000 ventilator-days in that period and applying from 2012 a package of verification to prevent VAP in children with mechanical ventilation in the pediatric intensive care unit.

RESULTS: Compliance with the parameters evaluated by VAP prevention verification package implemented in 2012 was $66 \%$ to $100 \%$, resulting in a decrease in the incidence of VAP of $47 \%$ in the period 2011-2012 and 69\% 2013, presenting rates VAP of 13.85, 7.29 and 4.3 pneumonias per 1,000 ventilator-days respectively.

CONCLUSIONS: The use of verification package for prevention of pneumonia is applicable and effective in reducing rates of VAP. This involves the commitment and multidisciplinary work of the whole service and a program to carry out this strategy of prevention to infections; the plan is implement this strategy in all intensive care units of the institution.

KEYWORDS: ventilator-associated pneumonia; VAP; bundles testing; prevention; pediatrics; effective prevention

\author{
${ }^{1}$ Médico adscrito al Departamento de \\ Infectología. \\ ${ }^{2}$ Maestro en Salud Pública. \\ ${ }^{3}$ Enfermera. \\ ${ }^{4}$ Jefe de Servicio de Unidad de Cuidados \\ Intensivos. \\ ${ }^{5}$ Medico pasante de Servicio Social. \\ Instituto Nacional de Pediatría, México.
}

\section{Correspondence}

Dra. Hilda Gpe. Hernández-Orozco

wuzhi1916@gmail.com

\section{INTRODUCCIÓN}

La neumonía asociada a ventilador es uno de los principales problemas de la Unidad de Cuidados Intensivos Pediátricos, con tasas de 13 neumonías por 1,000 días de ventilación mecánica, lo que implica estar por arriba del percentil 90\% reportado por la Red de Vigilancia Nacional de Salud (National Health Care Safety Network), quien reporta tasas de neumonías asociadas a ventilador por 1,000 días ventilador en hospitales pediátricos de entre 3 en hospitales de enseñanza y 7 en hospitales con pacientes neurológicos. ${ }^{1}$

Desde el 2013, la neumonía asociada a ventilador se define de acuerdo a los criterios clínicos del Centro de Prevención y Control de Enfermedades (CDC por sus siglas en inglés), como aquella infección que se presenta tres días después de la instalación de ventilación mecánica en el paciente y dos días después de presentar empeoramiento en la oxigenación $\mathrm{FiO}_{2}>0.20$ (20 puntos) del $\mathrm{FiO}_{2}$ mínimo diario en la basal e incremento de presión positiva al final de la espiración $\geq 3 \mathrm{~cm} \mathrm{H_{2 }} \mathrm{O}$ de la basal; además de los datos previos de cuadro clínico con alguno de los siguientes parámetros: fiebre $>38^{\circ} \mathrm{C}$ o hipotermia $<36.5^{\circ} \mathrm{C}$, en menores de un año de edad; tos, apnea, disnea, taquipnea, retracción de tórax, en menores de un año de edad; bradicardia $<100$ latidos por minuto o taquicardia >170 latidos por minuto; incremento de secreciones respiratorias o cambio en las características del esputo o de la necesidad de aspiración de secreciones, leucopenia $<4,000$ leucocitos $/ \mathrm{mm}^{3}$ o leucocitosis $>12,000$ leucocitos $/ \mathrm{mm}^{3}$. Además de radiografía de tórax con 
infiltrados nuevos o progresivos, consolidación, cavitación, neumatocele (en menores de un año). ${ }^{2}$ El instituto de mejora de la calidad (IHI por sus siglas en inglés) promueve el uso de paquetes de verificación como estrategia de mejora de la calidad. Dentro de ellos, el de prevención de neumonía asociada a ventilador evalúa y vigila que se cumplan los siguientes parámetros: la elevación de la cabeza del paciente, la higiene oral con clorhexidina, la evaluación diaria de disminución de sedación y extubación, profilaxis para úlcera péptica y profilaxis para trombosis venosa profunda. Aplicando esta estrategia en 35 unidades de cuidados intensivos de adultos se logró una disminución de $44.5 \%$ de las tasas de neumonía asociada a ventilador. ${ }^{3}$ Por ello, en nuestra institución se realiza este estudio piloto de aplicación de un paquete de verificación para prevenir neumonía asociada a ventilador en la Unidad de Terapia Intensiva Pediátrica.

\section{OBJETIVO}

Determinar la viabilidad y beneficios de aplicar un paquete de verificación para prevenir neumonía asociada a ventilador.

\section{MATERIAL Y MÉTODOS}

Estudio piloto transversal, ambispectivo. Se revisaron los datos de vigilancia epidemiológica de casos de neumonía asociada a ventilador, esto perteneció a resultados en 2011 en el que se reportaron 20; en 2012 doce y en 2013 catorce; se determinaron las tasas anuales de neumonía asociada a ventilador del periodo 2011 al 2013 al dividir el número de neumonías reportadas entre el denominador, días expuestos a ventilador de los pacientes de la unidad de cuidados intensivos de cada año: 1,444, 1,646 y 3,284, respectivamente y multiplicando el resultado por la base 1,000. En el 2012 se implementó un paquete de verificación en la Unidad de
Cuidados Intensivos Pediátricos en donde se evaluó la higiene de manos antes de realizar procedimientos; higiene oral con solución de clorhexidina y solución fisiológica a menores de 2 meses (o en caso de desabasto de solución de clorhexidina para los mayores de dos meses, situación extraordinaria) y cepillado dental; elevación de la cabeza; cambio de circuitos disfuncionales; evaluación diaria de retiro de ventilador; uso de profilaxis de úlcera de estrés; evaluación de disminución de la sedación y uso de sistemas cerrados para aspiración de secreciones; actividades realizadas por personal médico y de enfermería de la unidad de cuidados intensivos como parte de la atención del paciente con ventilación mecánica.

El seguimiento se realizó en 30 pacientes. La medición de exposición a ventilador mecánico en estos pacientes fue de 2,813 días ventilador; no se perdió ningún paciente durante el seguimiento. Los paquetes de verificación fueron aplicados durante la vigilancia epidemiológica por vocal de enfermería del comité de infecciones, en caso de observar que no se realizaba el procedimiento adecuado, se indicaba que se realizara. A la evaluación de retiro de ventilador se preguntaba al médico residente y la indicación de profilaxis gástrica se revisó en el expediente clínico. El estudio incluyó explorar el porcentaje del cumplimiento de las medidas recomendadas para la atención del paciente con ventilación mecánica y el cambio, ya que al aplicar un paquete de verificación se asegura que se lleven a cabo esas medidas, al recordar al personal que lo realizara en caso de omisión de alguna de ellas durante la observación.

Las observaciones sólo se realizaron en el turno matutino, durante la vigilancia epidemiológica diaria de rutina de la vocal de enfermería. La efectividad del paquete se midió mediante evaluación de la disminución de las tasas de neumonía asociada a ventilador por 1,000 días 
ventilador y el porcentaje de cumplimiento de las medidas evaluadas.

Para el análisis estadístico se utilizó el programa SPSS y se realizó análisis de frecuencias y cálculo de tasas por 1,000 días ventilador.

\section{RESULTADOS}

Se dio seguimiento a 30 pacientes observando todos los elementos del paquete en cada uno de ellos, siguiéndolos durante su condición de paciente con ventilación mecánica, retiro de ésta o egreso de la unidad. Los parámetros de elevación de la cabeza del paciente, higiene oral, evaluación de retiro de sedación y uso de equipo único (no reciclar material) se cumplieron en $100 \%$ de los eventos observados. Fue necesario indicar la corrección de conducta en la realización de higiene de manos, en donde sólo se realizó de primera intención en $66 \%$ en el año 2012 y $83 \%$ en el año 2013; el uso de equipo protector por personal durante la aspiración de secreciones y la evaluación de profilaxis para úlcera gástrica documentada fue de $66 \%$. La incidencia basal de neumonía asociada a ventilador de 2011 fue de 13.85 por 1,000 días-ventilador, disminuyendo a 7.29 por 1,000 días-ventilador en 2012, (año en que se implementó la estrategia de paquete de verificación), en el 2013 continuó la disminución con una tasa de 4.3 por 1,000 días-ventilador (Figura 1), lo que sugiere que la monitorización de los procedimientos mediante un paquete de verificación logra la disminución de la tasa de neumonías, ya que permite que el personal de los servicios de terapia intensiva e inhaloterapia vigilen cuidadosamente sus actividades. El uso de sistemas cerrados previsto no se logró de manera continua, por disminución de los recursos asignados, por lo que se excluyó del análisis.

\section{DISCUSIÓN}

La neumonía asociada a ventilador es la segunda causa de infecciones asociadas a la salud; repre-

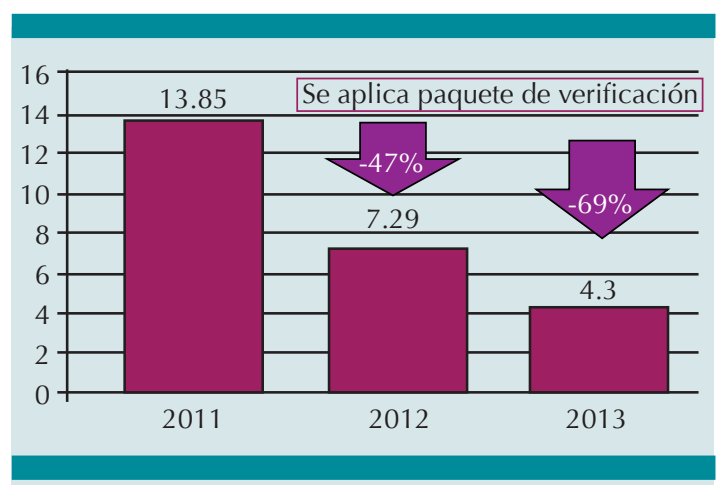

Figura 1. Disminución de tasas de neumonía asociada a ventilador por 1,000 días ventilador después de la implementación de un paquete preventivo de verificación.

senta $20 \%$ de las infecciones en la población y se reportan tasas de 6 a 11 neumonías por 1,000 días ventilador en Unidades de Cuidados Intensivos Pediátricos. ${ }^{4}$ La Red de Vigilancia Nacional de Salud refiere tasas entre 3 a 7 neumonías por 1,000 días ventilador, ${ }^{1}$ lo que subraya el problema que se presentó en nuestro hospital en el año 2011 con una incidencia de 13 neumonías por 1,000 días ventilador. El estudio de Elward y sus colaboradores refiere que los factores de riesgo identificados para desarrollar neumonía asociada a ventilador en adultos con duración de la ventilación mecánica, exposición a antibióticos, estancia hospitalaria prolongada y tratamiento con antiácidos bloqueadores de receptores de histamina $\mathrm{H} 2$; sin embargo, en este estudio ellos encontraron algunos otros factores de riesgo asociados a la población de la unidad de cuidados intensivos pediátricos: síndromes genéticos [OR 2.37 (IC 95\% 1.01-5.46)], reintubación [OR 2.71 (IC 95\% 1.18-6.21)] y el trasporte de pacientes fuera de la unidad [OR 8.9 (IC 95\% 3.8-20.7)]. ${ }^{4}$

Por otra parte, Munro y su equipo documentaron que desde 1983 el Centro de Prevención y Control de Enfermedades publicó una guía para prevención de neumonía asociada a ventilador, basada principalmente en recomendaciones de 
cómo realizar higiene de manos, manejo de medicamentos, equipo y fluidos respiratorios. En 2003 estas guías fueron modificadas y se recomienda la intubación orotraqueal versus nasotraqueal; el uso de ventilación no invasiva; la disminución de los días de ventilación mecánica y el cambio de circuitos sólo cuando se han contaminado o no funcionan. En 2005, el Instituto de Mejora de la Calidad, como parte de la campaña 100,000 vidas, implementó el uso de paquetes de verificación para la prevención de neumonía asociada a ventilador mediante la vigilancia adecuada de la higiene oral, posición con elevación de la cabeza, evaluación diaria de extubación, retiro o disminución de sedación, profilaxis versus úlcera de estrés y trombosis venosa profunda. ${ }^{5}$

Calvo y sus colegas ${ }^{6}$ hicieron una revisión del uso de medidas de prevención de neumonías y encontraron cuatro estudios en los que se documentó que el uso de paquetes de verificación disminuyó la incidencia de neumonías asociadas a ventilador por 1,000 días de ventilación mecánica de 6.6 a 2.7 (Resar R), de 8.2 a 3.3 (Berriel-Cass D), de 6.1 a 2.7 (Youngguist) y de 13.3 a 8.3 (Unahalekhaka), respectivamente. En este estudio, durante el primer año de aplicación del paquete de verificación, la incidencia de neumonía asociada a ventilador disminuyó $47 \%$ y el segundo año 69\% con disminución de la tasa basal de 13.85 a 4. ${ }^{3}$ En 2013, la disminución de la neumonía asociada a ventilador por 1,000 días/ventilador se generó gracias al compromiso y trabajo del personal de la unidad de cuidados intensivos que identificó los factores de riesgo e implementó actividades inmediata de mejora.

Así mismo, Calvo y sus colaboradores ${ }^{6}$ mencionaron que diferentes metanálisis y estudios concluyeron que las medidas de prevención con impacto en la disminución de neumonía asociada a ventilador fueron elevación de la cabeza [OR 0.47 (IC 95\% 0.27-0.82)], drenaje subglótico [RR 0.51 (IC 95\% 0.37-0.71)], descontaminación selectiva del tracto gastrointestinal en pacientes quirúrgicos [RR 0.19 (IC 95\% 0.15-0.26)] e higiene bucal con clorhexidina [RR 0.74 (IC 95\% 0.56-0.96)]; las medidas que no demostraron su eficacia fueron aspiración abierta versus cerrada [OR 0.96 (IC 95\% 0.76-1.21)], profilaxis de úlcera de estrés con bloqueadores H2 [OR 1.25 (IC 95\% 0.78-2)]. Estos resultados, de acuerdo con la evidencia científica, siguieren los puntos de un paquete de verificación a aplicar. Así, se recomienda se utilicen de preferencia, como máximo, cinco puntos para que la aplicación sea práctica. El paquete de verificación utilizado incluye los puntos desarrollados en las investigaciones antes descritas, con la deficiencia de que el uso de sistemas cerrados no pudo ser evaluado por problemas de insumos, situación que denota la importancia de gestionar los recursos necesarios para llevar a cabo la implementación de un paquete de verificación completo.

Un estudio que se realizó en una Unidad de Cuidados Intensivos Pediátricos menciona que la implementación de un paquete de prevención disminuyó la tasa de neumonía asociada a ventilador de 5.6 a 0.3 por 1,000 días/ventilador, mayor a lo que se encontró en el estudio de la institución, por lo cual se deben valorar los puntos supervisados en dicho paquete y determinar si debe ser implementado alguno a nuestro paquete de verificación. El paquete de verificación era similar al que se llevó a cabo en la institución e incluía: higiene de manos antes y después de la manipulación del ventilador, inspección de circuitos de ventilador y cambio cuando se encontraban sucios, drenaje de la condensación del circuito cada 4 horas o por turno, posición de la cabeza entre $30^{\circ}$ y $45^{\circ}$ y cuidado oral cada 4 horas. ${ }^{7}$

Este estudio fue una exploración para la aplicación de un paquete de verificación, lo que permitió detectar que la realización de procedimientos de acuerdo con las guías y manuales de 
procedimientos no se lleva a cabo en la rutina diaria. Además fue sorprendente que aún cuando las observaciones se realizaron en un número reducido de pacientes esto cambió la conducta del personal, ya que las desviaciones se observaron en el periodo inicial del estudio. La retroalimentación por el vocal de enfermería del comité al personal, el reforzamiento del jefe de servicio y del propio personal a sus compañeros de otros turnos sobre la necesidad de mejorar sus procedimientos se vio reflejada en la disminución de tasas de neumonía asociada a ventilador.

Las principales debilidades del estudio fueron el reducido número de pacientes, haber sido realizado sólo en turno matutino y que las observaciones se realizaran por vocal de enfermería del comité durante su vigilancia (cuando deberían ser llevados por personal del servicio). El análisis no permite determinar el cambio de conducta del inicio al final del estudio por las pocas observaciones realizadas. Como estudio piloto ésta es la primera documentación necesaria para determinar las fallas en el procedimiento rutinario de atención, por lo que se requiere realizar un estudio con menos deficiencias metodológicas. Aun así, demuestra que esta estrategia permite disminuir los índices de neumonía asociada a ventilador, por lo que se espera implementar de forma rutinaria y determinar con ello su beneficio real, como se menciona en la literatura.

\section{CONCLUSIÓN}

El uso de paquetes de verificación como medida preventiva ha sido ampliamente probado y éstos se deben aplicar en las Unidades de
Cuidados Intensivos para disminuir el riesgo de infecciones asociadas con la atención de la salud. En este estudio piloto se corroboró la necesidad de proponer su implementación en todas las Unidades de Cuidados Intensivos, de forma permanente, para determinar si la disminución observada en las tasas de neumonía asociada a ventilador se debe a su aplicación y puede ser una estrategia útil para disminuir su riesgo.

\section{REFERENCIAS}

1. Dudeck MA, Weiner LM, Allen-Bridson K, Malpiedi PJ, Peterson KD, Pollock DA, Sievert DM, Edwars JR. National Healthcare safety Network (NHSN) report, data summary for 2012, device-associated module. Am J Infect Control. 2013;41:1148-66.

2. July 2013 CDC/NHSN protocol corrections, clarification, and additions. DCD/NHSN definitions of healthcare-associated infections and criteria for type of specific infection in the care setting. Disponible en: http://www.cdc.gov/nhsn/ pdfs/validation/2013/pscmanual_july2013.pdf Accesado: enero 2016

3. Resar R, Griffin FA, Haraden C, Nolan TW, Using Care bundles to improve health care quality. IHI innovation series White paper. Canbridge, Massachusetts: Institute of Healthcare Improvement; 2012, p. 1-14. (Available on www.IHI.org).

4. Elward AM, Warren DK, Fraser VJ. Ventilator associated pneumonia in pediatric intensive care unit: riskfactors and outcomes. Pediatrics. 2002;109(5):758-64.

5. Munro N, Ruggiero M. Ventilator-associated pneumonia bundle. AACN Adv Crit Care. 2014;25(2):163-75.

6. Calvo MA, Delpiano LM, Chacón EV, Jemenao PI, Peña DA, Zambrano GA. Actualización consenso neumonía asociada a ventilación mecánica. Segunda parte. Prevención. Rev Chil Infect. 2011;28(4):316-32.

7. Bigham MT, Amato R, Bondurrat P, Fridriksson J, Krawczeski $C D$, et al. Ventilator-Associated Pneumonia in the Pediatric Intensive Care Unit: Characterizing the Problem and Implementing a Sustainable Solution. J Pediatrics. 2009;154(4):582-587e2. 